\title{
Supercontinuum Generation for Ultrahigh-Resolution OCT via Selective Liquid Infiltration Approach
}

\author{
Mojtaba SORAHI-NOBAR ${ }^{1}$, Alireza MALEKI-JAVAN ${ }^{2}$ \\ ${ }^{1}$ Dept. of Elect. Eng., South Tehran Branch, Islamic Azad University, Tehran, Iran \\ ${ }^{2}$ Dept. of Elect. Eng., Shahid Sattari University of Aeronautical Engineering, Tehran, Iran \\ st_m_sorahinobar@azad.ac.ir
}

Submitted June 8, 2017 / Accepted August 8, 2017

\begin{abstract}
In this paper, we apply liquid infiltration approach for supercontinuum generation (SCG) in photonic crystal fiber (PCF) in which by selectively infiltrating three air holes of PCF, a near zero dispersion is obtained that is a key parameter for SCG. Our numerical results show that by launching a very short input optical pulse of $50 \mathrm{fs}_{\mathrm{s}}$ in normal dispersion regime with wavelength centered about $700 \mathrm{~nm}$, into $50 \mathrm{~mm}$ PCF infiltrated by ethanol, broadband, coherent and ripple free SC as wide as $1000 \mathrm{~nm}$ will be achieved that covers the visible light and a part of near infrared spectra used for ultrahigh-resolution optical coherence tomography.
\end{abstract}

\section{Keywords}

Photonic crystal fiber (PCF), optical coherence tomography (OCT), liquid infiltration, supercontinuum generation, normal dispersion

\section{Introduction}

Optical coherence tomography (OCT) is one of the medical imaging methods for sensitive tissues such as eye and skin [1], [2]. The visible and near infrared light sources are used for scanning biological tissues. So, several conditions must be considered when selecting a light source for OCT imaging such as center wavelength, bandwidth, pulse shape and width, peak and average power [3]. Very high axial resolution images, which are provided by OCT, are independent of focusing conditions due to the different physical mechanisms determined the axial and lateral resolution [3]. Therefore, it is possible to enhance axial resolution by using broad bandwidth light sources. The light source not only determines axial OCT resolution via its bandwidth and central emission wavelength but also determines a penetration depth in the sample [4], [5]. A minimum output power with low amplitude noise is also necessary to enable high sensitivity and high speed with realtime OCT imaging. It is necessary to choose appropriate light sources in OCT structure due to the requiring of broadband light sources to increase the imaging resolution of spectroscopic OCT applications [6]. Recently, broad- band Ti:sapphire laser was developed in $5 \mu \mathrm{m}$ axial resolution spectroscopic OCT application [3]. Ultrahigh resolutions of 3 and $5 \mu \mathrm{m}$ were obtained at wavelengths of $800 \mathrm{~nm}$ and $1300 \mathrm{~nm}$ using continuum generation in a $100 \mathrm{~cm}$ PCF length with a parabolic dispersion profile with two closely spaced zero dispersion wavelengths [7]. Efforts have been made to reach a high resolution with supercontinuum (SC) source that is a high-intensity light that is a consequence of confining the light energy in both space and time [8-10]. It is a broadband and coherent light that is generated via the interaction of an optical short pulse with a nonlinear media [11], [12]. With this light source, resolution of images is much more than other light sources and even OCT imaging with submicron resolution can be obtained. Humbert et al. reported flat and broad SCG using $100 \mathrm{~cm}$ PCF. It provides $1.5 \mu \mathrm{m}$ axial resolution in free space at $809 \mathrm{~nm}$ center wavelength [13]. Ghanbari et al. demonstrated an ultrahigh resolution OCT system using PCF to produce a high axial resolution in biological tissues [4]. Povazay et al. experimentally demonstrated that using $6 \mathrm{~mm}$ long PCF pumped by a $50 \mathrm{fs}$ optical pulse, 0.75 and $0.5 \mu \mathrm{m}$ axial resolution in a free space and biological tissue could be achieved, respectively [14]. Champert et al. demonstrated the simultaneous excitation of PCF for SCG in visible and near-infrared range for OCT application [15]. It needs to couple two signals simultaneously into a PCF which is relatively hard. Kudlinski et al. experimentally demonstrated SCG for OCT with a spectrum covering the region from $470 \mathrm{~nm}$ to $1750 \mathrm{~nm}$ by using a $300 \mathrm{~m}$ long GeO2-doped-core PCF with decreasing ZDW along its length [16]. Some researchers used dispersion engineering by means of varying the PCF structural parameters, like air holes diameter, the lattice constant, and the number of the air-hole rings to achieve flat dispersion in a wide wavelength range for SCG [17], [18]. This technique depends on the technological capability to realize a specific design with high precision. Precision in controlling the geometrical parameters during the fabrication process is the most critical constraint in acquiring the desired dispersion [19], [20]. Nonetheless, the use of liquid infiltration approach as a post-fabrication technique enables one to tailor PCFs properties as desired, avoiding the topological limitations. both sides of the infiltrated holes are sealed with silica to prevent liquid from evaporating [11], [18]. 
This paper is focused on the SC generation through PCF with a liquid infiltration approach for white light and OCT application. Selective infiltration of air-holes by linear optical liquids is used to control and tailor the PCFs' dispersion profiles, as desired. This PCF has an important role in the OCT setup by increasing the resolution and improving the quality of tissue images. On the other hand, the average power required in the proposed PCF is less than other methods of OCT imaging that solves the thermal requirements [21]. Other considerations such as loss, effective area, and nonlinear coefficient are taken into account.

\section{Physical Structure of PCF}

Figure 1 shows the perspective and cross-sectional views of solid core PCF consisting of circular air-holes (gray circles) with diameter of $d=0.9 \Lambda$ in background of silica (blue circle). Red circles in the innermost ring represent the selectively infiltrated air-holes, by the appropriately chosen optical liquid in which the refractive indices of Silica, Water, Ethanol, Chloroform, and Carbon tetrachloride as a function of wavelength are given by Sellmeier equation as

$$
n(\lambda)=\left\{1+B_{1} \lambda^{2}\left(\lambda^{2}-C_{1}\right)^{-1}+B_{2} \lambda^{2}\left(\lambda^{2}-C_{2}\right)^{-1}\right\}^{0.5}
$$

where $B_{i}$ and $C_{i}\left(\mu \mathrm{m}^{2}\right)(i=1,2)$, in Sellmeier equation for different used materials are presented in Tab. 1. The air holes are arranged in a triangular lattice in eight hexagonal rings with pitch size of $\Lambda=1.1 \mu \mathrm{m}$ and core diameter $d_{\mathrm{C}}=2 \Lambda-d=1.2 \mu \mathrm{m}$. In the next section, we will show that this infiltration results in a low loss, flat and low dispersion, and highly nonlinear PCF suitable for generating the supercontinuum spectra. Moreover, experimental techniques for filling one or more specific air-holes in a PCF have already been developed [22].
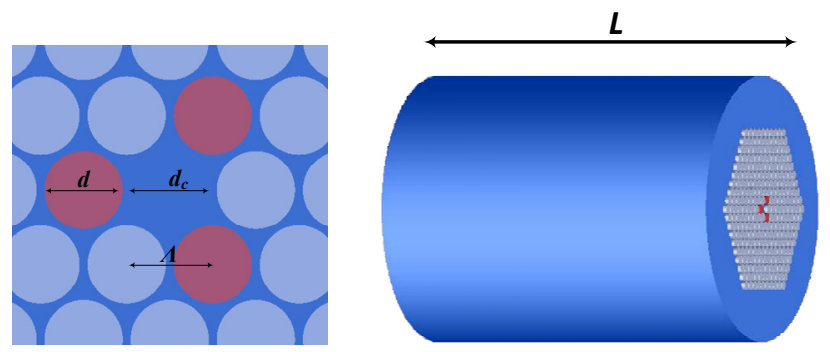

Fig. 1. Left: a cross-sectional view of PCF. Right: Perspective view of PCF.

\begin{tabular}{|c|c|c|c|c|}
\hline \multirow{2}{*}{ Material } & \multicolumn{4}{|c|}{ Sellmeier coefficients } \\
\cline { 2 - 5 } & $B_{1}$ & $C_{1}\left(\mu \mathrm{m}^{2}\right)$ & $B_{2}$ & $C_{2}\left(\mu \mathrm{m}^{2}\right)$ \\
\hline Silica & 0.68 & 0.004 & 0.42 & 0.013 \\
\hline Water & 0.75 & 0.01 & 0.085 & 8.91 \\
\hline Ethanol & 0.83 & 0.009 & -0.15 & -49.45 \\
\hline Chloroform & 1.04 & 0.01 & 0.003 & 0.15 \\
\hline Carbon tetrachloride & 1.09 & 0.011 & - & - \\
\hline
\end{tabular}

Tab. 1. Constants $B_{i}$ and $C_{i}\left(\mu \mathrm{m}^{2}\right)(i=1,2)$, in Sellmeier equation for different used materials.

\section{Theory and Calculations}

\subsection{Mathematical Background}

In this paper, to demonstrate the propagation of optical pulse inside the PCF shown in Fig. 1, we have considered nonlinear Schrödinger equation (NLSE). Other researchers demonstrated that the numerical results calculated by this model are close to the experimental results [12], [23-25]. Evolution of an optical pulse, having a slowly varying electric field amplitude represented by envelope function $\mathrm{A}(z, t)$ defined by GNLSE [24]:

$$
\begin{aligned}
& \frac{\partial A}{\partial z}+\frac{\alpha}{2} A+\sum_{n=1}^{8} i^{(n-1)} \frac{\beta_{n}}{n !} \frac{\partial^{n} A}{\partial t^{n}}= \\
& i\left(\gamma\left(\omega_{0}\right)+i \gamma_{1} \frac{\partial}{\partial t}\right) \cdot A(z, t) \int_{-\infty}^{t} R\left(t^{\prime}\right)\left|A\left(z, t-t^{\prime}\right)\right|^{2} \mathrm{~d} t^{\prime}
\end{aligned}
$$

where $z$ and $t$ are the spatial coordinates along the fiber and the time variable, $\alpha$ is total fiber loss, $\beta_{n}$ is the $n^{\text {th }}$ dispersion order that in our calculations, second to eighth orders of dispersion are considered, $\gamma$ is the nonlinear coefficient where $\gamma=n_{2} \omega_{0} / c A_{\text {eff }}\left(\omega_{0}\right)$ in which $\mathrm{A}_{\text {eff }}$ is the effective area of the fundamental mode, $\omega_{0}$ is the pulse radial frequency, $c$ is speed of light and $n_{2}$ is the nonlinear Kerr index which is $2.7 \times 10^{-20} \mathrm{~m}^{2} / \mathrm{W}$ for silica as a background material of the fiber. $R(t)$ is the response function including the Raman and Kerr nonlinearities. Assuming that the electronic contribution is nearly instantaneous, the functional form of $R(t)$ can be written as:

$$
R(t)=\left(1-f_{\mathrm{R}}\right) \delta(t)+f_{\mathrm{R}} h_{\mathrm{R}}(t)
$$

where $\delta(t)$ is the Dirac delta function, $f_{\mathrm{R}}$ represents the fractional contribution of the delayed Raman response function $h_{\mathrm{R}}(t)$, which in turn takes an approximate analytic form of

$$
h_{\mathrm{R}}(t)=\frac{\tau_{1}^{2}+\tau_{2}^{2}}{\tau_{1} \tau_{2}^{2}} \exp \left(\frac{-t}{\tau_{2}}\right) \sin \left(\frac{t}{\tau_{1}}\right)
$$

with $f_{\mathrm{R}}=0.18, \tau_{1}=12.2$ fs and $\tau_{2}=32 \mathrm{fs}$, for silica.

\subsection{PCF Engineering}

Since we intend to generate SC in the range from visible to near infrared, we have to engineer zero dispersion wavelength (ZDW) of the PCF. One of these approaches is changing the core diameters. Reducing the core diameter has some limitations: first, PCF fabrication is hard to do, second, while propagating in fibers, short wavelengths can pass through small core cross section area but long wavelengths that require larger effective cross-sectional area cannot be confined in the core and they might enter the cladding [24]. This will be considered as a leakage loss because it is not accessible anymore. We used the liquid infiltration technique to resolve this challenge. To demonstrate the role of liquid infiltration, we compare the 

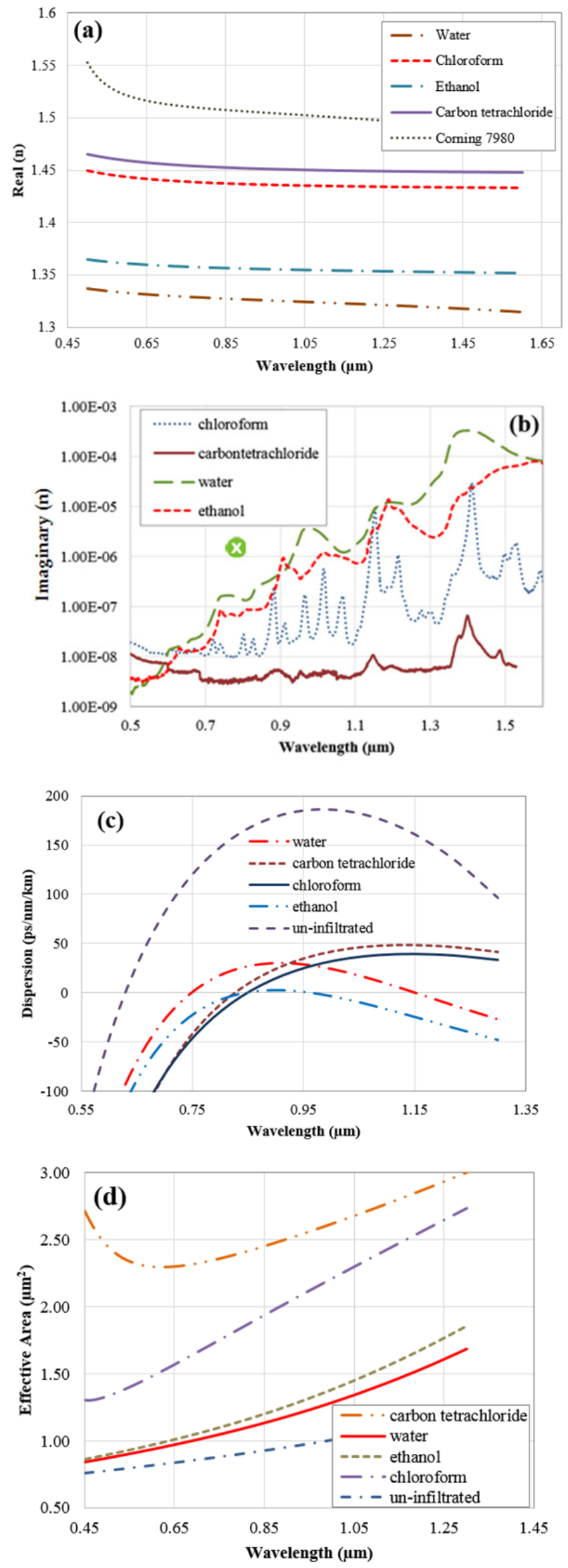
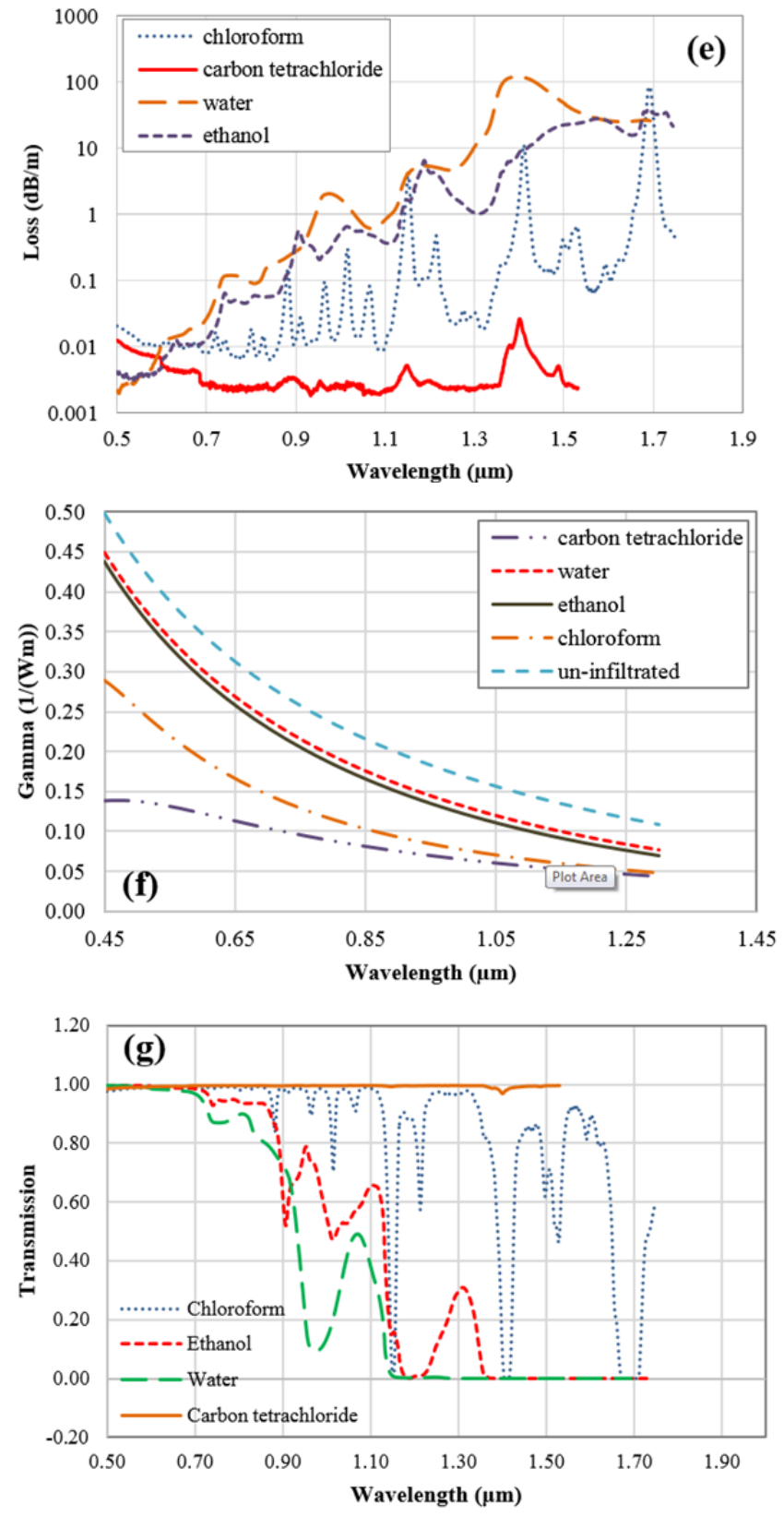

Fig. 2. Comparison of the (a) real part, (b) imaginary part of the liquid refractive indices, (c) total loss, (d) effective mode area, (e) dispersion profiles and (f) nonlinear parameter of the un-infiltrated PCF with those selectively infiltrated with optical liquids of various indices, and $(\mathrm{g})$ transmission profiles [24].

dispersion, $D(\lambda)$, effective area, $A_{\text {eff }}(\lambda)$, total loss, $L_{\mathrm{t}}(\lambda)$, and nonlinear coefficient, $\gamma(\lambda)$, of un-infiltrated and infiltrated fibers in Fig. 2. We have set our goal in this section to investigate the possibility of engineering these profiles, as desired, via selective liquid infiltration. It is already demonstrated that by infiltrating three air holes of the PCF's innermost ring with an appropriate liquid, depicted by the red circles in Fig. 1, the dispersion profile can be engineered as desired.

The real and imaginary parts of the refractive indices of water, ethanol, chloroform and carbon tetrachloride used in this paper versus wavelength are shown in Fig. 2(a), (b). 
As can be observed in Fig. 2(a), the refractive index of silica is more than all mentioned liquids. Therefore, the guiding mechanism is provided by the total internal reflection along the solid core fiber.

Dispersion is calculated as a function of wavelength in the range of $0.55-1.35 \mu \mathrm{m}$. It is shown in Fig. 2(c) for the PCF infiltrated with four mentioned liquids having well-known losses.

The ZDW for un-infiltrated PCF equals to $0.63 \mu \mathrm{m}$ and the dispersion gradient near the ZDW is about $10 \mathrm{ps} /\left(\mathrm{nm}^{2} \cdot \mathrm{km}\right)$. Figure 2(c) illustrates when the air holes are infiltrated by liquids, ZDW is shifted toward longer wavelengths, and the dispersion slope is decreased. The most and the least significant ZDW shifts are observed for chloroform and water about 0.85 and $0.75 \mu \mathrm{m}$, respectively. By shifting ZDW using the liquid infiltration approach as desired, we can use available lasers with femtosecond optical pulses as input sources.

Dispersion is the key profile for SC generation but there are some other linear and nonlinear parameters for the occurrence of nonlinear phenomena such as the small effective area of the PCF. PCF infiltration results in increasing the effective area that implies nonlinearity reduction.

Figure 2(d) shows our calculations of the effective area as a function of wavelength in the range of $0.45 \mu \mathrm{m}$ to $1.3 \mu \mathrm{m}$ for the PCF filled with the liquids mentioned before. As can be observed in this figure, the effective area is $0.858 \mu \mathrm{m}^{2}$ at $0.7 \mu \mathrm{m}$ for the un-infiltrated case.

When the PCF is infiltrated with any liquid, the effective area increases. The smallest increase is detected for water $\left(1.01 \mu \mathrm{m}^{2}\right)$, and the largest one is for carbon tetrachloride $\left(2.322 \mu \mathrm{m}^{2}\right)$. It is clear that the increase of the effective area is relatively small that can be ignored for nonlinear applications.

Liquid infiltration introduces significant loss in transmission, despite the fact that the core is all solid and we only consider the fundamental mode. The imaginary part of the refractive index represented in Fig. 2(b) may have more effect than the real part of the guiding properties.

The attenuation caused by the liquid infiltration restricts the benefits resulting from the improvement of the dispersion. It is calculated as a function of wavelength for the PCF infiltrated with the liquids. The results are plotted in Fig. 2(e) that shows the loss increases with increasing of wavelength.

The maximum loss is observed for the PCF infiltrated with water, for instance, it is $0.026 \mathrm{~dB} / \mathrm{m}$ for $0.7 \mu \mathrm{m}$. The minimum loss is observed for carbon tetrachloride that is $0.002 \mathrm{~dB} / \mathrm{m}$ for $0.7 \mu \mathrm{m}$. The loss at this level does not limit the $\mathrm{SG}$ because $\mathrm{SG}$ is achieved at a few cm long.

Figure 2(f) depicts nonlinear coefficient versus wavelength in the range of 0.45 to $1.3 \mu \mathrm{m}$. The maximum and minimum nonlinear coefficients are for un-infiltrated and carbon tetrachloride respectively. Because in these two cases, the effective area is lower and higher than the others, respectively.

Figure 2(g) shows the transmittance curves as a function of wavelength for $5 \mathrm{~cm}$ thickness in $20^{\circ} \mathrm{C}$ for used liquids in this paper. It demonstrates that carbon tetrachloride has the maximum transmission window. However, water has the minimum one.

\section{Results and Discussions}

So far, we have learned how to engineer and control the linear and nonlinear properties of a PCF over a wide wavelength range via selective liquid infiltration.

In this section, using the computed parameters and other necessary detailed information, we investigate how to generate SC using the liquid infiltration approach over a piece of PCF. By solving NLSE in both time and frequency domains, we show the propagation of optical pulse along the engineered PCF. In this numerical study, we assumed optical pulse of peak power $P_{0}=50 \mathrm{~kW}$ with a width of $T_{0}=50 \mathrm{fs}$ and wavelength of $\lambda_{0}=700 \mathrm{~nm}$, are launched into $50 \mathrm{~mm}$ length of PCF shown in Fig. 1. Due to this short length, the total loss reported in [26] for silica PCF is ignored. Our simulations reveal that the leakage power inside infiltrated holes is insignificant and can be neglected in the rest of the paper.

Figure 3 compares the spectral distributions of the optical pulses evolving along the un-infiltrated PCF with those obtained from selectively infiltrated PCFs. Results show spectrum in the un-infiltrated case has severe ripples, which are not desirable. On the other hand, considering the noise, the ripple will be more intense. Figure 3 illustrates the potential of the liquid infiltration technique for SC generation with flat bandwidth, simply by choosing an appropriate liquid and the input signal wavelength. As can be observed in this figure, the maximum spectral broadening occurs for water and ethanol respectively. In the case of water, SC is wider than other liquids because it has the least value of refractive index among presented liquids in this study that leads to PCF having the ZDW closer to the wavelength of the input pulse. As you see, the archived spectrum by water infiltration does not have enough intensity in some wavelengths with several ripples. But ethanol has enough bandwidth and intensity and also too much coherence because according to [27], $\mathrm{n}$ wavelength of $700 \mathrm{~nm}$, the forth order of dispersion, $\beta_{4}$, is positive with a value more than other used liquids in this paper at the same wavelength. Therefore, we will use ethanol as our desired infiltrating liquid for OCT applications in the following. It is clear that by increasing the optical pulse peak power, broader SC is achieved. Various linear and nonlinear phenomena are accountable for the generation of the supercontinua observed in Fig. 3. In the case of ethanol shown in Fig. 3(c), when pumping 50 fs pulses in the normal dispersion regime at $700 \mathrm{~nm}$, the pulses initially undergo strong self-phase modulation (SPM), possibly leading to optical wave breaking (OWB) due to self-steepening 
(SS) and third-order dispersion (TOD), which causes a significant part of the light to be blue-shifted. The redshifting part will eventually cross the ZDW, at which point soliton dynamics, in particular Raman induced soliton selffrequency shifting (SSFS), will dominate further spectral broadening. As expected, we observed distinct SPM broadening close to the $700 \mathrm{~nm}$ pump, and soliton dynamics when crossing the ZDW. These dynamics were remarkably well reproduced by simulating propagation of only the fundamental mode [25]. These phenomena can be done for other cases of liquids. Figure 3(a) shows launching input optical pulse inside un-infiltrated case in anomalous dispersion regime not only doesn't generate SC but also reduces the imaging resolution caused by many ripples in the output spectrum.

As can be seen in Figs. 3(b)-4(e), depending on the refractive index of the infiltrated liquid, SC with desired intensity and bandwidth is achieved. With this technique, we
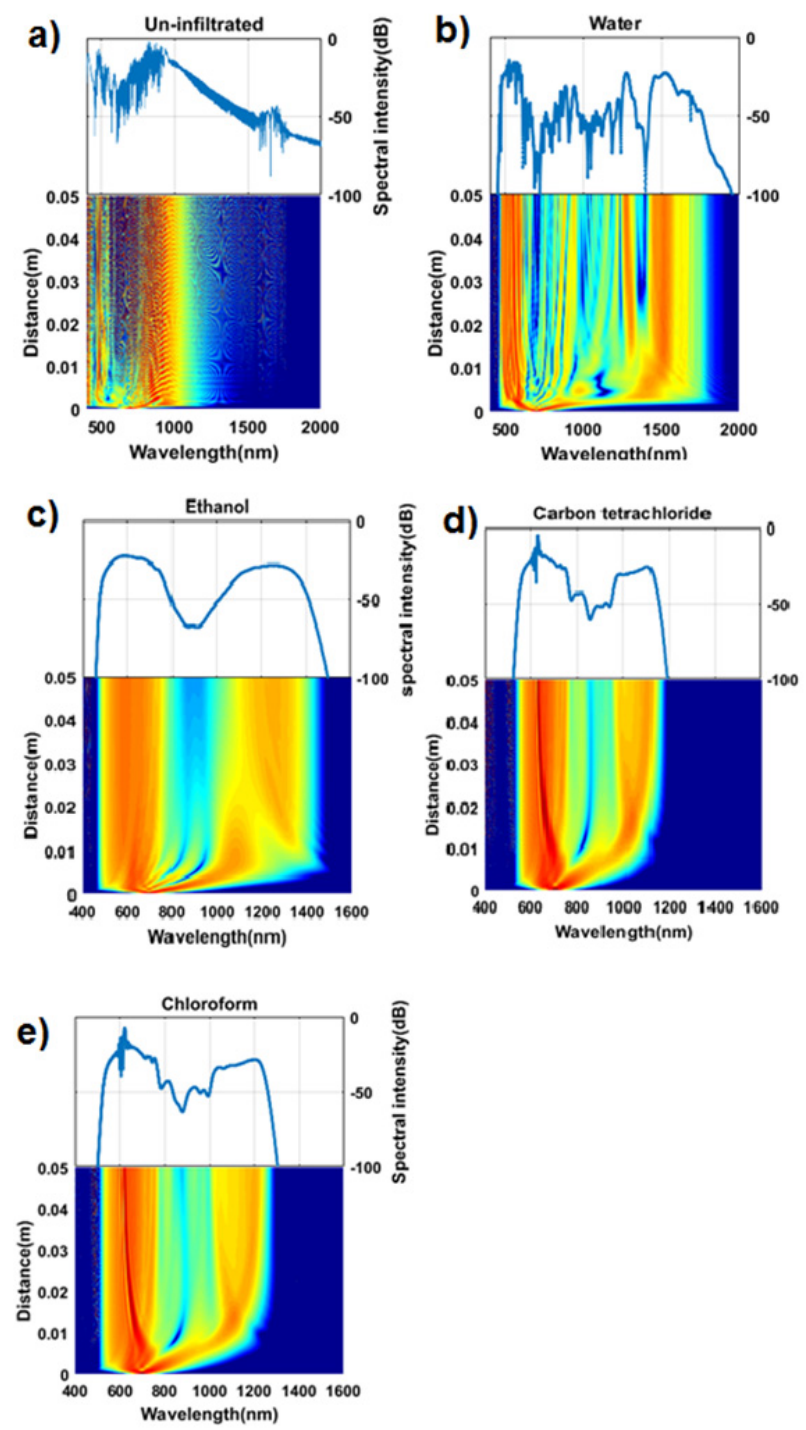

Fig. 3. Evolution of the spectral distribution of optical pulses of 50 -fs widths $P_{0}=50 \mathrm{~kW}$ peak power, along $50 \mathrm{~mm}$ long un-infiltrated PCFs, compared with those obtained from selectively infiltrated PCFs with optical liquids of various refractive indices. don't need to change the physical parameters of the PCF such as air hole diameter, core diameter and lattice constant by too complex fabrication processes. The optical liquids used for this purpose must be available and transparent to the wavelengths of interest. However ethanol as a suitable liquid used in this paper is flammable that the infiltration process should be done carefully. So, available liquids help us implement OCT setup so easier, faster and cheaper and also changing the infiltrated liquids leads to adjust the ZDW in the range of available laser sources. Consequently, by the appropriate choice of the infiltrated liquid, input optical pulse and PCF properties, a broad and coherent spectrum at the fiber output is achieved. Figure 3(c) demonstrates that a coherence SC as wide as $1040 \mathrm{~nm}$ is generated at $50 \mathrm{~mm}$ length of the PCF infiltrated with ethanol in which the imaging resolution via this fiber is about $0.2 \mu \mathrm{m}$. It is very small and ideal compared to values obtained from recent articles and can highly increase the resolution of medical images. With this technique, axial resolution increases largely, and more detailed information of the tissue is considerable. It means that the detection of the disease is so easier and faster.

\section{Conclusion}

We have studied the generation of supercontinua in visible and near-infrared frequency ranges, utilizing silicabased solid core PCF with the liquid infiltration approach. Simulations demonstrated when a 50 fs input optical pulse of peak power $50 \mathrm{~kW}$ and center wavelength $700 \mathrm{~nm}$ are launched into a $50 \mathrm{~mm}$ long PCF infiltrated by ethanol, spectral broadening as wide as $1040 \mathrm{~nm}$ in both visible and near-infrared regions can be obtained. The broad bandwidth of the light source permits high imaging resolution in which this PCF as a source of OCT system can generate tissues images with a longitudinal resolution of $0.2 \mu \mathrm{m}$. This fact can significantly help faster and more accurate detection of tissue diseases.

\section{References}

[1] SCHUMAN, J. S., PUliafito, C. A., Fujimoto, J. G., et al. Optical Coherence Tomography of Ocular Diseases. $3^{\text {rd }}$ ed. Thorofare, NJ: SLACK Inc., 2013. ISBN: 13 978-1-55642-864-7

[2] AVANAKI, M. R., CERNAT, R., TADROUS, P. J., et al. Spatial compounding algorithm for speckle reduction of dynamic focus OCT images. IEEE Photonics Technology Letters, 2013, vol. 25, no. 15, p. 1439-1442. DOI: 10.1109/LPT.2013.2266660

[3] DREXLER, W., FUJIMOTO, J. G. (Eds.) Optical Coherence Tomography: Technology and Applications. Springer Science \& Business Media, 2015.ISBN: 978-3-319-06418-5

[4] GHANBARI, A., KASHANINIA, A., SADR, A., et al Supercontinuum generation for optical coherence tomography using magnesium fluoride photonic crystal fiber. OptikInternational Journal for Light and Electron Optics, 2017, vol. 140, p. 545-554. DOI: 10.1016/j.ijleo.2017.04.099

[5] IKUNO, Y., MARUKO, I., YASUNO, Y., et al. Reproducibility of retinal and choroidal thickness measurements in enhanced depth imaging and high-penetration optical coherence tomography. 
Investigative Ophthalmology \& Visual Science, 2011, vol. 52, no. 8, p. 5536-5540. DOI: $10.1167 /$ iovs.10-6811

[6] UNTERHUBER, A., POVAZAY, B., BIZHEVA, K., et al. Advances in broad bandwidth light sources for ultrahigh resolution. Physics in Medicine and Biology, 2004, vol. 49, no. 7, p. $1235-1246$

[7] AguirRe, A., NiShiZAWA, N., FUJimOTO, J., et al. Continuum generation in a novel photonic crystal fiber for ultrahigh resolution optical coherence tomography at $800 \mathrm{~nm}$ and 1300 nm. Optics Express, 2006, vol. 14, no. 3, p. 1145-1160. DOI: 10.1364/OE.14.001145

[8] DIOUF, M., SALEM, A. B., CHERIF, R., et al. Super-flat coherent supercontinuum source in As 38.8 Se 61.2 chalcogenide photonic crystal fiber with all-normal dispersion engineering at a very low input energy. Applied Optics, 2017, vol. 56, no. 2, p. 163-169. DOI: 10.1364/AO.56.000163

[9] SAGHAEI, H. Supercontinuum source for dense wavelength division multiplexing in square photonic crystal fiber via fluidic infiltration approach. Radioengineering, 2017, vol. 26, no. 1, p. 16-22. DOI: $10.13164 / \mathrm{re} .2017 .001$

[10] SAGHAEI, H., MORAVVEJ-FARSHI, M. K., EBNALIHEIDARI, M., et al. Ultra-wide mid-infrared supercontinuum generation in As 40 Se 60 chalcogenide fibers: solid core PCF versus SIF. IEEE Journal of Selected Topics in Quantum Electronics, 2016, vol. 22, no. 2, 8 p. DOI: 10.1109/JSTQE.2015.2477048

[11] EBNALI-HEIDARI, M., SAGHAEI, H., KOOHI-KAMALI, F., et al. Proposal for supercontinuum generation by optofluidic infiltrated photonic crystal fibers. IEEE Journal of Selected Topics in Quantum Electronics, 2014, vol. 20, no. 5, 8 p. DOI: 10.1109/JSTQE.2014.2307313

[12] SAGHAEI, H., EBNALI-HEIDARI, M., MORAVVEJ-FARSHI, M. K. Mid-infrared supercontinuum generation via As 2 Se 3 chalcogenide photonic crystal fibers. Applied Optics, 2015, vol. 54, no. 8, p. 2072-2079. DOI: 10.1364/AO.54.002072

[13] HUMBERT, G., WADSWORTH, W., LEON-SAVAL, S., et al. Supercontinuum generation system for optical coherence tomography based on tapered photonic crystal fibre. Optics Express, 2006, vol. 14, no. 4, p. 1596-1603. DOI: 10.1364/OE.14.001596

[14] POVAZAY, B., BIZHEVA, K., UNTERHUBER, A., et al. Submicrometer axial resolution optical coherence tomography. Optics Letters, 2002, vol. 27, no. 20, p. 1800-1802. DOI: 10.1364/OL.27.001800

[15] CHAMPERT, P.-A., COUDERC, V., LEPROUX, P., et al. Whitelight supercontinuum generation in normally dispersive optical fiber using original multi-wavelength pumping system. Optics Express, 2004, vol. 12, no. 19, p. 4366-4371

[16] KUDLINSKI, A., BOUWMANS, G., VANVINCQ, O., et al. White-light cw-pumped supercontinuum generation in highly $\mathrm{GeO}$ 2-doped-core photonic crystal fibers. Optics Letters, 2009, vol. 34, no. 23, p. 3631-3633.DOI: 10.1364/OL.34.003631

[17] EBNALI-HEIDARI, M., DEHGHAN, F., SAGHAEI, H., et al. Dispersion engineering of photonic crystal fibers by means of fluidic infiltration. Journal of Modern Optics, 2012, vol. 59, no. 16 , p. 1384-1390.

[18] SAGHAEI, H., HEIDARI, V., EBNALI-HEIDARI, M., et al. A systematic study of linear and nonlinear properties of photonic crystal fibers. Optik-International Journal for Light and Electron
Optics, 2016, vol. 127, no. 24, p. 11938-11947. DOI: 10.1016/j.ijleo.2016.09.111

[19] COEN, S., CHAU, A. H. L., LEONHARDT, R., et al. White-light supercontinuum generation with 60-ps pump pulses in a photonic crystal fiber. Optics Letters, 2001, vol. 26, p. 1356-1358. DOI: 10.1364/OL.26.001356

[20] SAITOH, K., KOSHIBA, M., HASEGAWA, T., et al. Chromatic dispersion control in photonic crystal fibers: application to ultraflattened dispersion. Optics Express, 2003, vol. 11, p. 843-852. DOI: 10.1364/OE.11.000843

[21] FERCHER, A., DREXLER, W., HITZENBERGER, C., et al Optical coherence tomography - principles and applications. Reports on Progress in Physics, 2003, vol. 66, no. 2, p. 239-303.

[22] NIELSEN, K., NOORDEGRAAF, D., SøRENSEN, T., et al. Selective filling of photonic crystal fibres. Journal of Optics A: Pure and Applied Optics, 2005, vol. 7, no. 8, p. L13-L20. DOI: $10.1088 / 1464-4258 / 7 / 8 / \mathrm{L} 02$

[23] DUDley, J. M., GENTY, G., COEN, S. Supercontinuum generation in photonic crystal fiber. Reviews of Modern Physics, 2006, vol. 78, p. 1135-1184. DOI: 10.1103/RevModPhys.78.1135

[24] PNIEWSKI, J., STEFANIUK, T., VAN, H., et al. Dispersion engineering in nonlinear soft glass photonic crystal fibers infiltrated with liquids. Applied Optics, 2016, vol. 55, no. 19, p. 5033-5040. DOI: 10.1364/AO.55.005033

[25] HORI, T., NISHIZAWA, N., GOTO, T., et al. Experimental and numerical analysis of widely broadened supercontinuum generation in highly nonlinear dispersion-shifted fiber with a femtosecond pulse. Journal of the Optical Society of America B, 2004, vol. 21, no. 11, p. 1969-1980. DOI: 0.1364/JOSAB.21.001969

[26] FROSZ, M. H., SøRENSEN, T., BANG, O. Nanoengineering of photonic crystal fibers for supercontinuum spectral shaping. Journal of the Optical Society of America B, 2006, vol. 23, no. 8, p. 1692-1699. DOI: 10.1364/JOSAB.23.001692

[27] BARH, A., GHOSH, S., VARSHNEY, R. K., et al. An efficient broad-band mid-wave IR fiber optic light source: design and performance simulation. Optics Express, 2013, vol. 21, no. 8, p. $9547-9565$. DOI: $10.1364 /$ OE. 21.009547

\section{About the Authors ...}

Mojtaba SORAHI-NOBAR was born in London in 1987. $\mathrm{He}$ received M.Sc degree in Electrical Engineering from the Dept. of Electrical Engineering, South Tehran Branch, Islamic Azad University, Tehran, Iran in 2017. His research interests are nonlinear photonic crystal fibers and waveguides, nonlinear optics and optical communications.

Alireza MALEKI-JAVAN received M.Sc and Ph.D degrees in Electrical Engineering from K.N. Toosi University of Technology, Tehran, Iran in 2000 and 2009, respectively. Now, he is with the Dept. of Electrical Engineering, Shahid Sattari Aeronautical University of Science and Technology. His current research interests include nonlinear photonic crystal structures and terahertz waves. 